\title{
An Efficient Data Transmission in VANET Using Clustering Method
}

\author{
C. Suganthi Evangeline and S.Appu
}

\begin{abstract}
A special type of Mobile Ad-hoc Networks (MANETs) which has frequent changes of topology and higher mobility is known as Vehicular Ad-hoc Networks (VANETs). In order to divide the network into groups of mobile vehicles and improve routing, data gathering, clustering is applied in VANETs. A stable clustering scheme based on adaptive multiple metric combining both the features of static and dynamic clustering methods is proposed in this work. Based on a new multiple metric method, a cluster head is selected among the cluster members which is taken from the mobility metrics such as position and time to leave the road segment, relative speed and Quality of Service metrics which includes neighborhood degree, link quality of the RSU and bandwidth. A higher QoS and cluster stability are achieved through the adaptive multiple metric. The results are simulated using NS2 and shows that this technique provides more stable cluster structured with the other methods.
\end{abstract}

Keywords-VANET, RSU, 2-layer stable clustering scheme.

\section{I.INTRODUCTION}

$\mathbf{V}$ EHICULAR Ad hoc Networks (VANET) provides critical information about roads and traffic condition. It sends safety messages and entertains passengers. The abundant on-board processing resources of the vehicles is an unique feature of VANET, compared to other nodes. This helps in processing complex algorithms for different applications.

Lot of research have been conducted on VANETs in the last few years, regarding routing techniques and data dissemination. Target tracking, where the on-board vehicle sensors are used is a main application entertaining the passengers. Vehicles keep entering and leaving the cluster, selection of proper metrics for cluster head election and cluster membership in a highly dynamic environment such as VANET is a challenge.

Two different approaches for the clustering of vehicles are used in VANET: 1. static clustering which is based on Vehicle-to-Infrastructure (V2I) communication in which Road Side Units (RSUs) act as static cluster heads. The vehicles are essentially connected with RSUs In order to have real-time communication and connectivity with the Internet. Sometime, due to the large distance between the RSUs, the vehicles with high mobility are not always connected to the RSUs. A simple VANET architecture is shown in fig 1.1. 2. Dynamic clustering which is based on Vehicle-to-Vehicle (V2V) communication, here cluster heads are chosen from cluster

C. Suganthi Evangeline is with Karunya University the department of Electronics and Communication Engineering (e-mail: evangelineme4 @ gmail.com)

S. Appu is with Karunya University, Communication Engineering (e-mail: appu7033@gmail.com). members. This method alters the concept of static cluster heads. The vehicles either join or leave the clusters according to their speed to identified cluster heads as the dynamic clusters are in motion on the road. The V2V communications are more flexible and independent of the roadside conditions in this case. These algorithms lack in quality of service even though they have cluster stability and overhead minimization.

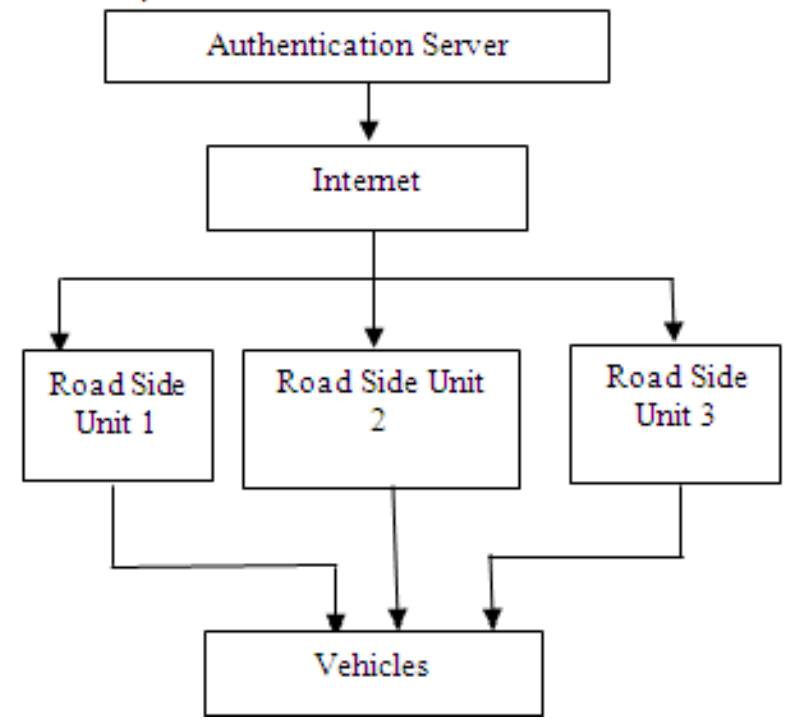

Figure 1 VANET architecture

So a Dynamic Backbone Assisted (DBA) MAC protocol is presented to support Geocast communication on highway scenarios. This is a new cluster-based protocol proposed to prolong the network lifetime which contains a distributed clustering algorithm providing stability and channel quality of each link considering the mobility of vehicles these algorithms form the stable clusters and maintain the stability during communications and link failures and satisfies the Quality of Service requirements. During the formation of clusters a tradeoff between the Quality of Service and the mobility metrics should be taken into consideration in order to improve the stability of the network,

To achieve this a new clustering approach is proposed to provide stability, scalability and QoS for the cluster. On Comparing with previous schemes requirements of the vehicular applications and the quality of link to the RSU is taken into account during the cluster creation process. With the simulation results, we find that, the approach which is proposed is more stable and can support RSU-based applications better (e.g. Internet). 
Sybil attack is explained as a malicious entity that can present multiple identities at once. This is One of the direct means through the third entity is convinced by the other two that they are distinct is to run, at the same time, some tasks that one entity cannot do it alone. Many techniques have been proposed such as testing resources based on computational and communication challenges in order to ensure the identity of a node. The Sybil attack is a dangerous attack in a VANET environment with disastrous consequences. As we know VANET supports the information transmission between vehicles, the rear-end collision between vehicles, and the warning about dangerous situations in real time. For example the disguised attacker sends wrongly the messages such as the information transmission between vehicles, the rear-end collision between vehicles, and the warning about dangerous situations, the other vehicles suffer a confusion. Sybil attack can be a serious threat to a VANET's function on causing the other vehicles change the route on the road or leave the road for the attacker. If any node creates multiple copies of itself, then it creates confusion in the network claiming all the authority thus resulting in network collision. This is known as Sybil attack in the network. This can attack both internally and externally in which external attacks. Therefore it is found that Sybil attack is by sending multiple messages from different ids. It is generally of two types delay sensitive and throughput sensitive.

\section{III.CLUSTERING METHODS FOR VANETS}

Clustering is useful in network management and data aggregation in complex distributed and large scale networks. An aggregator node or leader node $(\mathrm{CH})$ is introduced which is responsible for data aggregation in a specific part of the network based on the characteristics of vanet. The role of this node is to build and maintain the cluster structure for communication to decrease congestion in both V2V and V2I communications reducing the number of messages being transmitted within the network and provides optimal quality of service (QoS), to avoid link breakage and density variations. This decreases contention and also hidden terminal problems. A special benefit of clustering in VANET environment is that. It deals with the dynamic topology of VANETs and also adapts to changes that occur in topology. Here, the whole network is divided into smaller parts that are less dynamic than the global network. This is due to the lesser relative mobility between the nodes in the network. The main objective is to choose the best appropriate nodes which has more similar mobility patterns in order to join the same cluster. This also reduces channel contention and provides fair channel access, which increases network capacity by controlling the topology. Many schemes based on dividing the network into clusters and using inter cluster communication are introduced using clusterbased techniques to reduce the effect of handoff latency in VANETs and minimizes the packet loss caused by handoff.

\section{A. Efficient features and stability of the cluster}

There are several performance metrics which measures cluster stability. To create more stable and robust clustering protocols that can perform proper functions in VANET's highly dynamic environment and also can adapt to frequent changes in topology and density has been the main objective of all the clustering protocols. The main stability and efficiency features considered in most clustering algorithms are given below. To design implement an efficient and stable clustering algorithm improvement in these features can be taken into account.

\section{B. Clustering Stability and Efficiency Improvement in VANET}

After many researches on increasing clustering efficiency and stability of the clusters in VANET, it is found that designing efficient clustering protocols with high cluster stability is a challenging task because of the dynamic nature of VANET, since it requires novel techniques and ideas. Some of the common methods used in many VANET clustering algorithms are listed as the follows:

(i) Appropriate $\mathrm{CH}$ selection metric:

(ii) Appropriate cluster membership rules

(iii) Reduction of $\mathrm{CH}$ changes

(iv) Association of nodes to cluster instead of $\mathrm{CH}$

(v) $\quad \mathrm{CH}$ Recovery Techniques

(vi) Overhead reduction technique

\section{IV.WORK DONE}

In order to overcome this Sybil attack we go for clustering method. In situations where in the number of vehicles is less and the identities are more, the delay and throughput is higher. As the traffic is more, this clustering method results in better efficiency while it reduces delay and throughput. When DSDV protocol is carried out alongside clustering method, the possibility of Sybil attack is further reduced. This is because the number of vehicles are already observed by the cluster head.

\section{RESULTS AND DISCUSSION}

The simulation is carried out using the NS2 simulation tool and the following results are obtained.

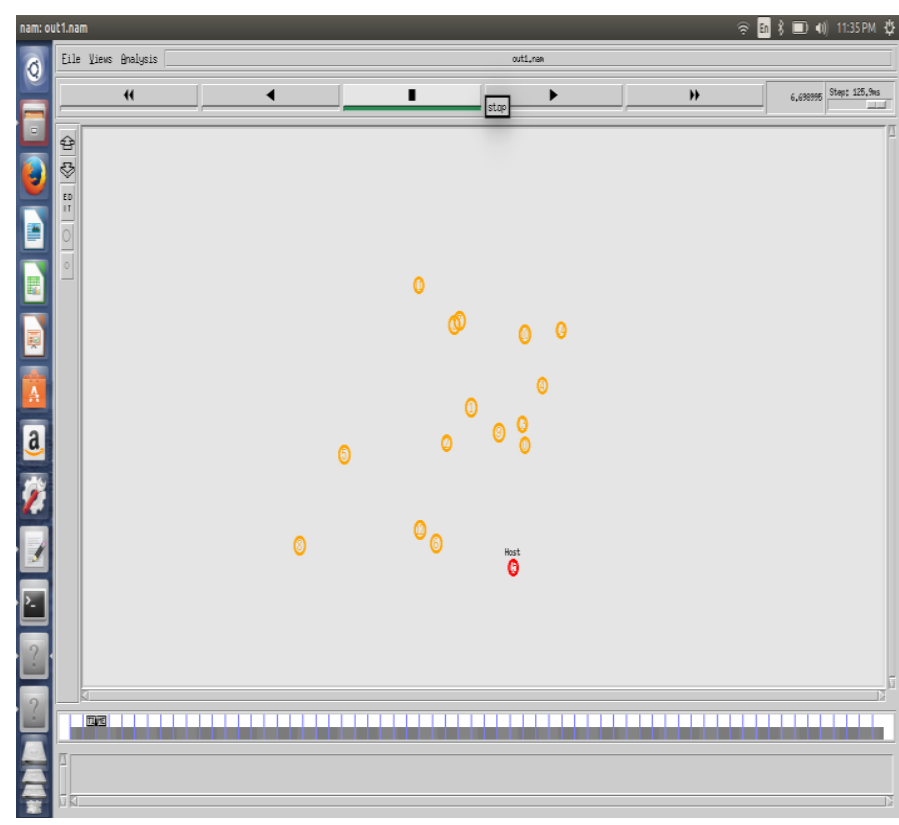

Figure 2 Clustering of nodes 


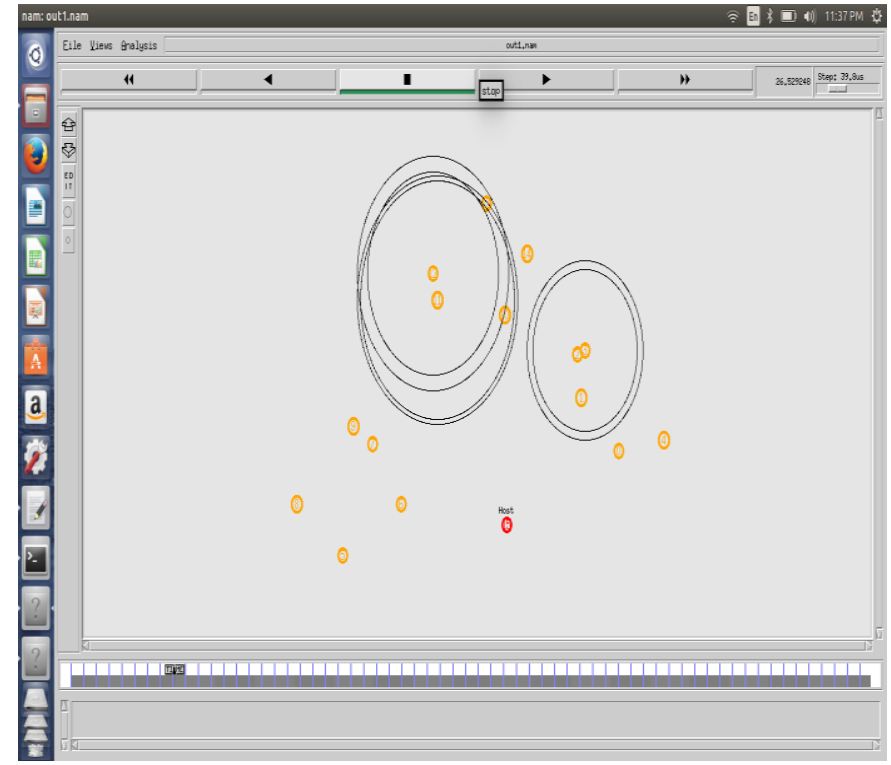

Figure 3 Formation of clusters

Figure 2 represents the number of nodes which is used for the formation of clustering of the nodes.

Figure 3 represents the grouping of the nodes in clusters where the transmission of packets are done.

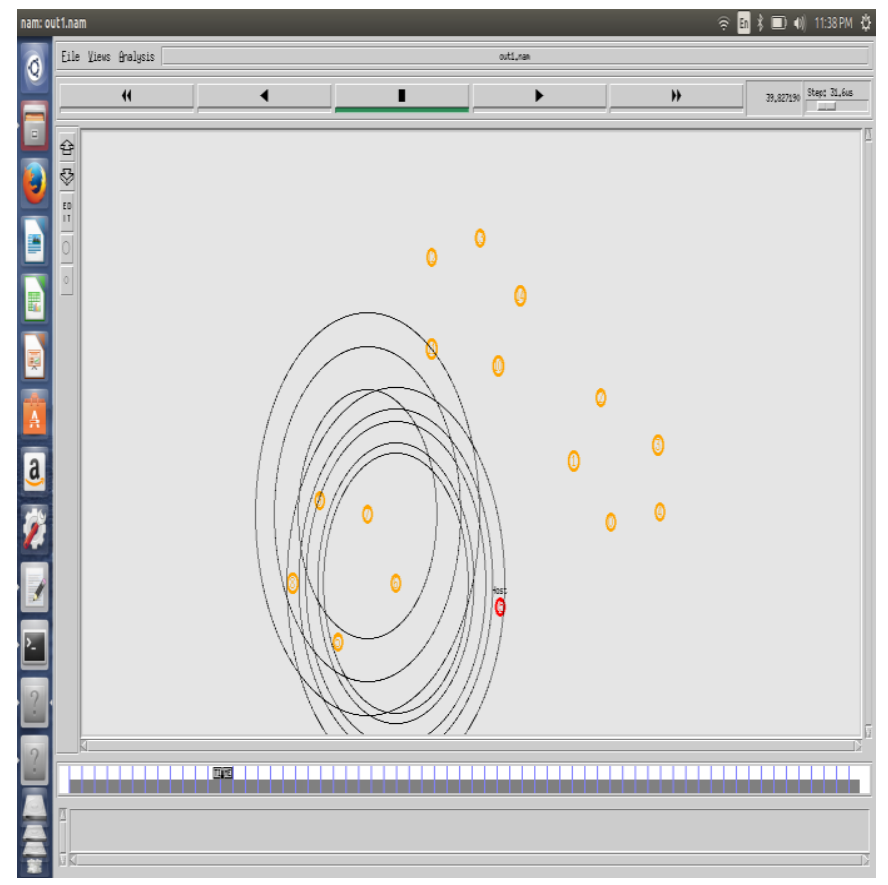

Figure 4 Transmission of packets in clusters

Figure 4 represents the transmission of packets only in the clusters and also with the host node.

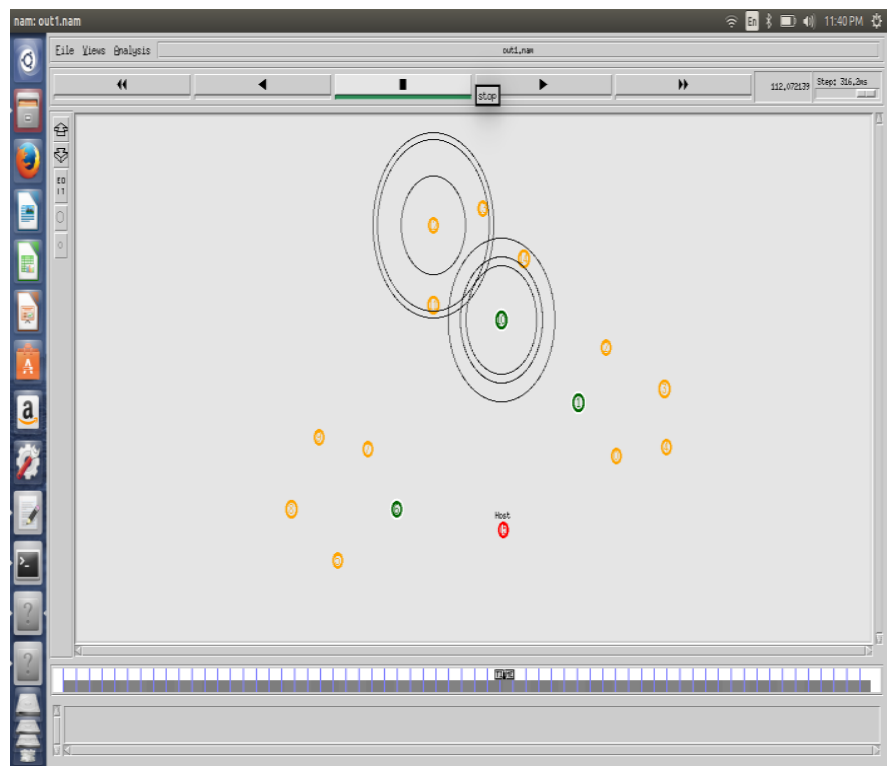

Figure 5 Formation of Cluster Head

Figure 5 represents the formation of the cluster in the clustering nodes and also the transmission of packets from head to host

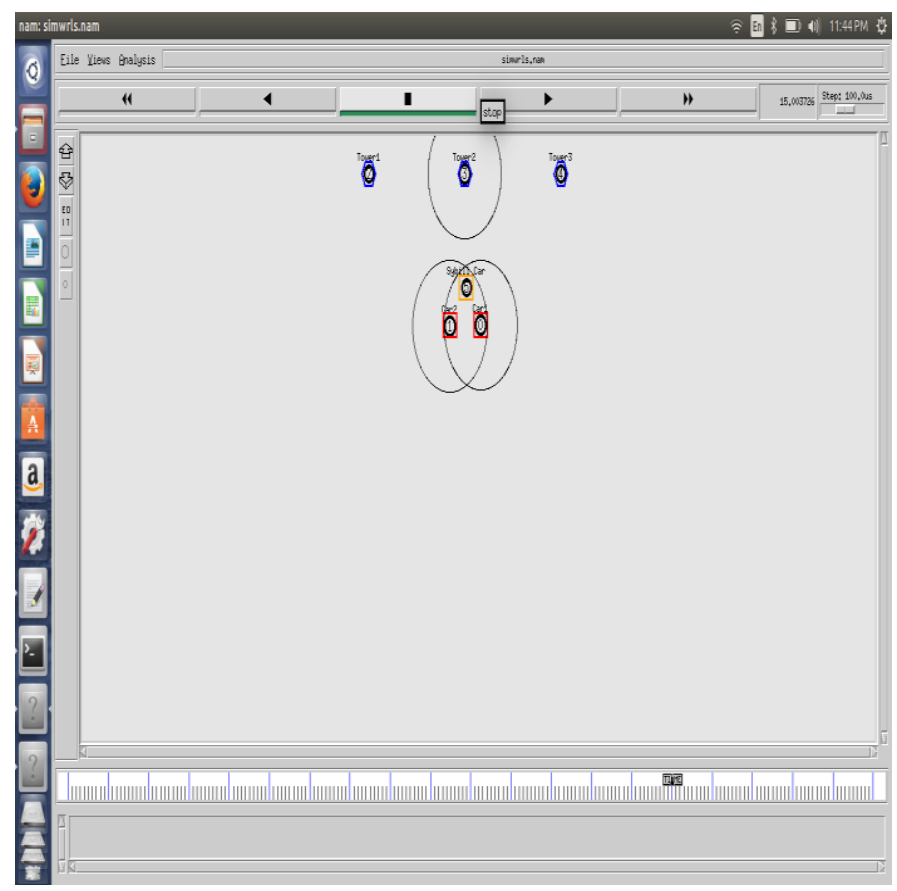

Figure 6 Nodes in Sybil attack

Figure 6 represents the Sybil attack car where the multiple nodes are transferred from the single node 


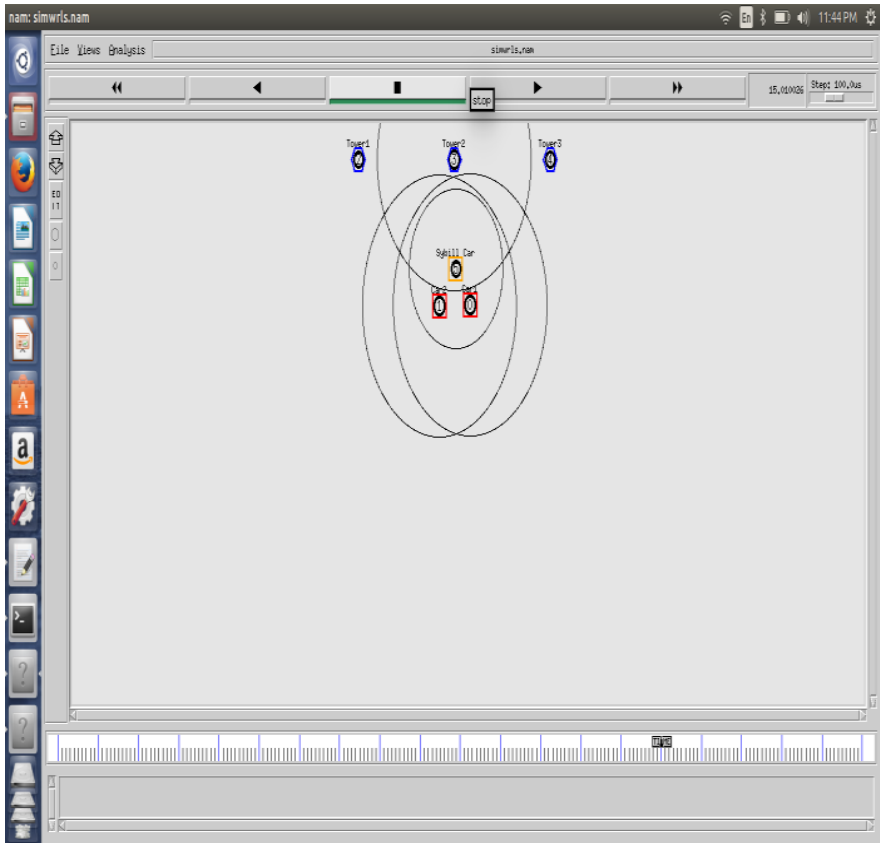

Figure 7 Formation of Sybil attack

Figure 7 represents the formation of the Sybil attack with the tower 2.

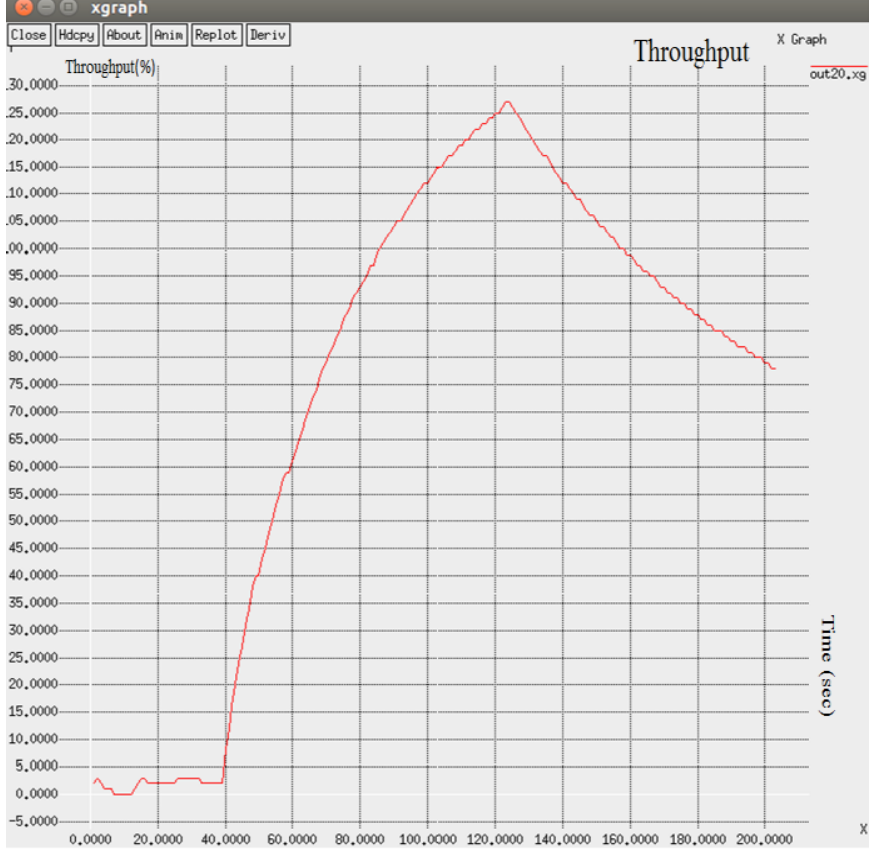

Figure 8 Throughput for clustering

Figure 8 represents the change in the throughput where time increases, a peak is formed and then the throughput is reduced when time increases.

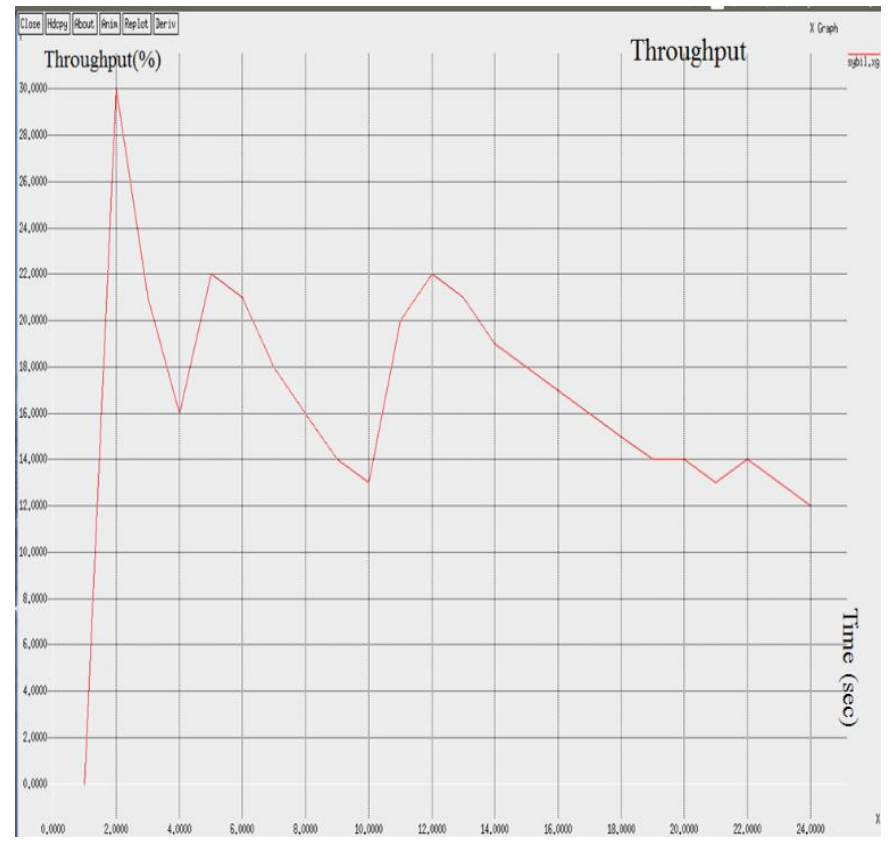

Figure 9 Throughput for Sybil attack

Figure 9 represents the throughput for the Sybil attack where number of packets delivered is more in traffic, throughput increases to a peak and then decreases and increases mildly with respect to time.

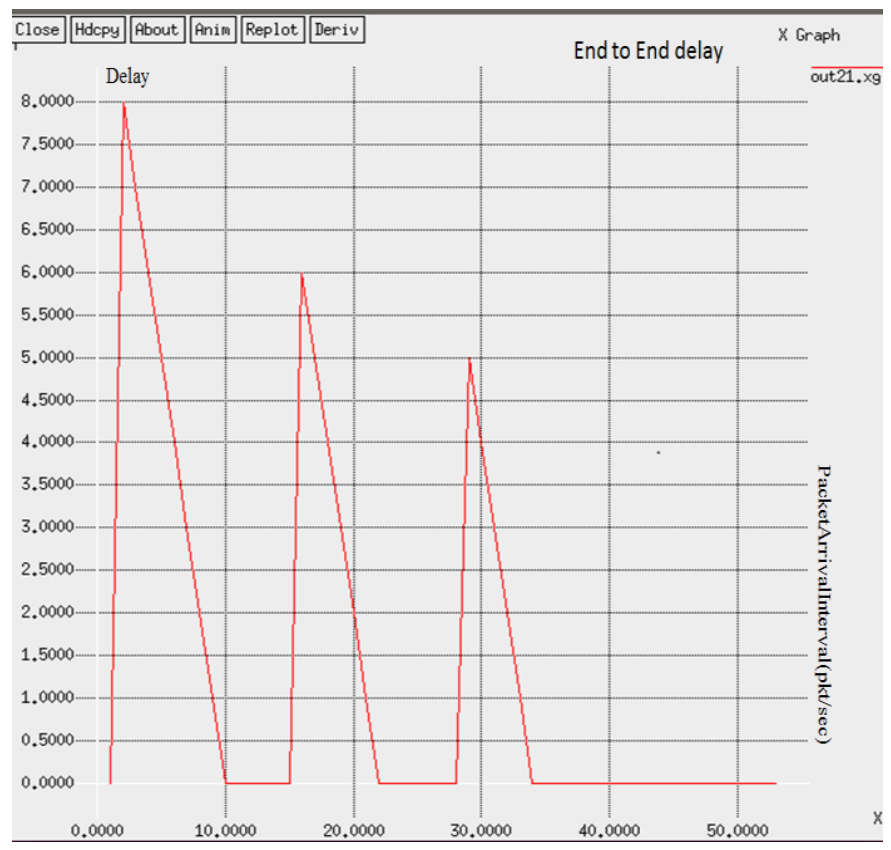

Figure 10 Delay in clustering of nodes

Figure 10 represents the delay in clustering of nodes where the delay is decreased with respect to the packet arrival interval. 


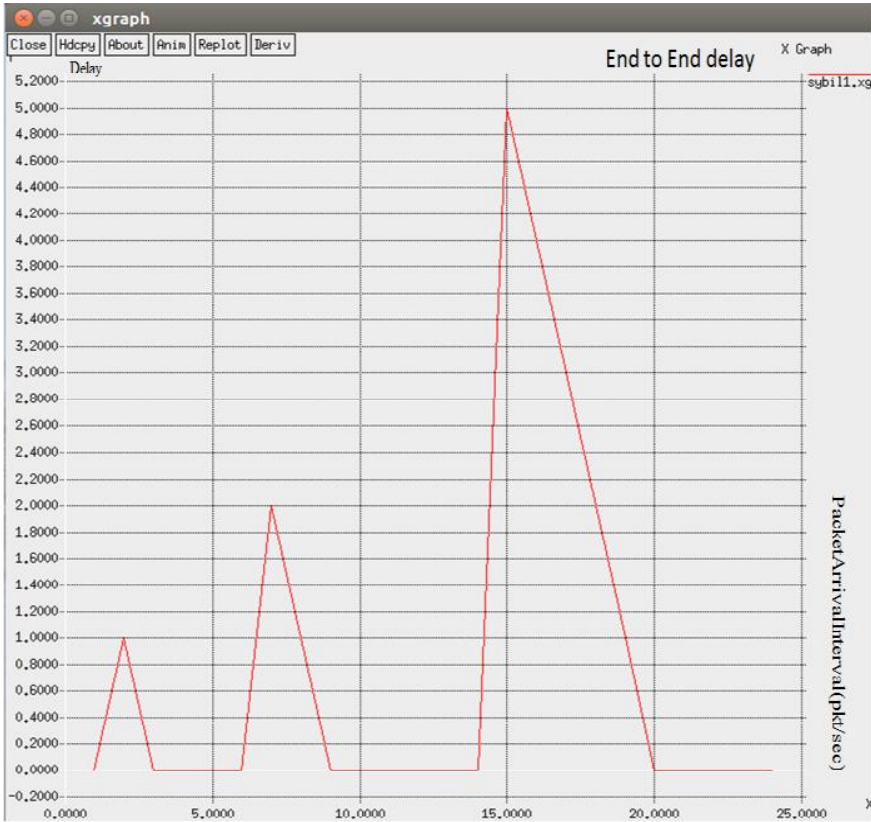

Figure 11 Delay in Sybil attack

Figure 11 represents the delay in Sybil attack where the delay is increased with respect to the packet arrival interval.

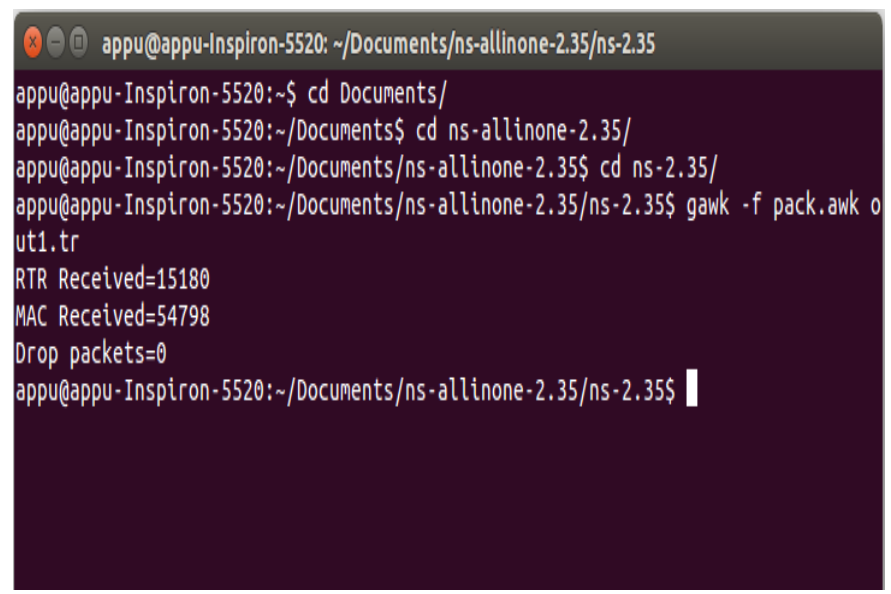

Figure 12 Packet delivery ratio in clustering

Figure 12 represents the number of RTR and MAC packets sent during clustering and also the number of packets dropped. Figure 13 represents the number of TCP and ACK packets send during the Sybil attack where packet delivery ratio is calculated.

\section{CONCLUSION}

One of the biggest issue in VANET is security. In order to detect the attacker's presence in the Route discovery phase. The proposed technique uses the clustering method to overcome Sybil attack which leads to a secure communication network on detection of Sybil nodes. Through this method the overall performance and efficiency of the network which in turn results in increased throughput and lesser delay. The clusters which are created here are stable. It also exhibits a long average cluster member duration, long average cluster head duration, and low average rate of cluster head change.

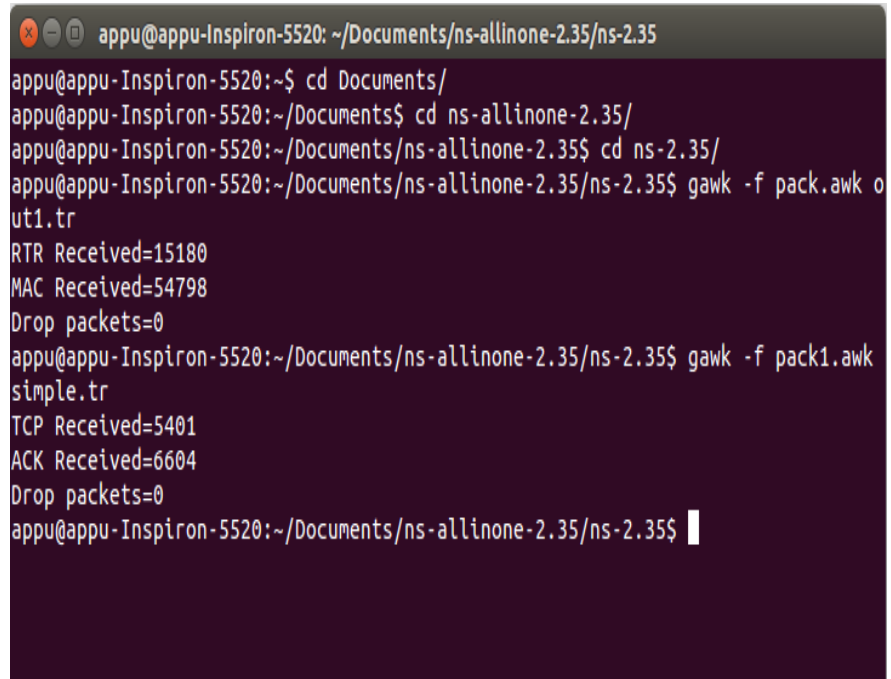

Figure 13 Packet delivery ratio in Sybil attack

\section{REFERENCES}

[1] Agarwal, T. Little "Access point placement in vehicular networking”, in Proc. IEEE Wireless Access for Vehicular Environments (WAVE) 2008, Dearborn, MI. December 2008.

[2] A.Wasef, Y. Jiang, and X. Shen, "ECMV: efficient certificate management scheme for vehicular networks", Proc. IEEE Globecom'08, New Orleans, LA, USA, Nov. 30 - Dec. 4, 2008.

[3] B. Parno and A. Perrig, "Challenges in securing vehicular networks", in Prof. of the Workshop on Hot Topics in Networks (HotNets-IV) 2005 College Park, Maryland, November 2005.

[4] C. Jung, C. Sur, Y. Park, and K. Rhee, "A robust conditional privacy preserving authentication protocol in VANET", in Proc. MobiSec 2009, pp. 35-45, Turin, Italy, June 2009.

[5] C. Lochert, B. Scheuermann, C. Wewetzer, A. Luebke, and M. Mauve, "Data aggregation and roadside unit placement for a vanet traffic information system", in Proc. the fifth ACM international workshop on VehiculAr Inter-NETworking 2008, San Francisco, California, USA September 15, 2008 .

[6] E. Coronado and S. Cherkaoui, "Service discovery and service access in wireless vehicular networks," in Proc. IEEE Transaction 2008, pp. 1-6.

[7] F. El Ali and B. Ducourthial, "A light architecture for opportunistic vehicle-to-infrastructure communications," in Proc. MobiWac, Bodrum, Turkey, Oct. 2010, pp. 60-67.

[8] J. Zhao and G. Cao, "VADD: Vehicle-assisted data delivery in vehicular ad hoc networks", IEEE Transaction on Vehicular Technology, 2008. 57(3): p. 1910-1922.

[9] J. Zhao, T. Arnold, Y. Zhang and G. Cao, "Extending drive-thru data access by vehicle-to-vehicle relay", IEEE Transaction on Vehicular Technology, 2008. 57(3): p. 1910-1922.

[10] M. Raya and J. P. Hubaux, "Securing vehicular ad hoc networks", Journal of Computer Security, Vol. 15, No. 1, pp. 39-68, 2007.

[11] R. Lu, X. Lin, H. Zhu, P.-H. Ho and X. Shen, "ECPP: efficient conditional privacy preservation protocol for secure vehicular communications", in Proc. INFOCOM 2008, pp. 1229-1237, 2008.

[12] X. Dong, L. Wei, H. Zhu, Z. Cao, and L. Wang, "EP2DF: An efficient privacy-preserving data-forwarding scheme for service-oriented vehicular ad hoc networks," IEEE Transaction on Vehicular Technology., vol. 60, no. 2, pp. 580-591, Feb. 2011.

[13] X. Lin, X. Sun, P. H. Ho, and X. Shen, "GSIS: a secure and privacy preserving protocol for vehicular communications", IEEE Transaction on Vehicular Technology, Vol. 56, No. 6, pp. 3442-3456, 2007.

[14] Y. Peng, Z. Abichar, and J. M. Chang, "Roadside-aided routing (RAR) in vehicular networks", in Proc. IEEE ICC 2006, Vol. 8, pp. 3602-3607, Istanbul, Turkey, June 2006 